\section{Aspectos generales de etiología y tratamiento de la sinusitis crónica}

General aspects of etiology and treatment of chronic sinusitis

Michelle Stefanía Miranda Cevallos

Médico general, Clínica Zymasalud,

michumiky@gmail.com.

https://orcid.org/0000-0002-5872-

236X. Guayaquil, Ecuador

Paola Alejandra Herrera Herrera

Médico general, Centro Médico

Club de Leones Quito, palolita-

ldu@hotmail.com,

https://orcid.org/0000-0002-6756-

8106. Guayaquil, Ecuador

Cesar Emilio Vargas Baños

Especialista en Administración

Hospitalaria, Hospital General del

Norte de Guayaquil Los Ceibos,

cesarvargasmd1@gmail.com,

https://orcid.org/0000-0001-5231-

3373 Guayaquil, Ecuador

http://www.jah-

journal.com/index.php/jah

Journal of American health

Julio - Diciembre vol. 3. Num. 2 - 2020

Esta obra está bajo una Licencia

Creative Commons

Atribución-NoComercial-

CompartirIgual 4.0 Internacional.

RECIBIDO: 8 DE FEBRERO 2019

ACEPTADO: 11 DE AGOSTO 2019

PUBLICADO: 2 DE JULIO 2020

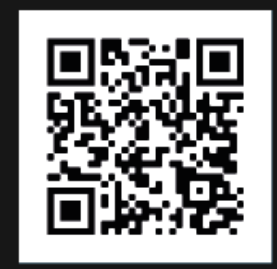

Scan this QR

code with your

smart phone or

mobile device to

read more papers

\section{RESUMEN}

La sinusitis crónica es una enfermedad crónica que implica inflamación a largo plazo de la mucosa del seno nasal y paranasal. A pesar de su prevalencia generalizada y su impacto sustancial en la población, no existen medicamentos aprobados para su manejo y tratamiento. Esto se debe en gran medida a la falta de investigación sobre tratamientos seguros y efectivos, que a su vez está relacionado con la falta de comprensión de la fisiopatología de la enfermedad. El objetivo de este articulo proporcionar una revisión de la literatura sobre los referentes teóricos de la sinusitis crónica, con la finalidad de actualizar y profundizar los conocimientos sobre su etiología y de los aspectos generales del tratamiento clínico o quirúrgico que sean de utilidad para la comunidad científica. Se analizaron 20 artículos de revisión de la literatura y 5 artículos de análisis de datos sobre el tema. Se recopilaron artículos desde el año 2010 al 2019, tanto en idioma inglés como en español. La evidencia respalda la irrigación salina diaria de alto volumen con terapia tópica con corticosteroides como terapia de primera línea para la sinusitis crónica. Se puede considerar un ciclo corto de corticosteroides sistémicos (1-3 semanas), ciclo corto de doxiciclina ( 3 semanas) o un antagonista de leucotrienos en pacientes con pólipos nasales. Se puede considerar un ciclo prolongado (3 meses) de antibiótico macrólido para pacientes sin pólipos.

PALABRAS CLAVE: sinusitis crónica, rinosinusitis, diagnóstico, tratamiento, antifúngico, anfotericina $\mathrm{B}$. 


\section{ABSTRACT}

Chronic sinusitis is a chronic disease that involves long-term inflammation of the nasal and paranasal sinus mucosa. Despite its widespread prevalence and its substantial impact on the population, there are no approved medications for its management and treatment. This is largely due to a lack of research on safe and effective treatments, which in turn is related to a lack of understanding of the pathophysiology of the disease. The objective of this article is to provide a review of the literature on the theoretical references of chronic sinusitis, in order to update and deepen the knowledge about its etiology and the general aspects of clinical or surgical treatment that are useful for the scientific community. Twenty literature review articles and 5 data analysis articles on the subject were analyzed. Articles were collected from 2010 to 2019, in both English and Spanish. Evidence supports high-volume daily saline irrigation with topical corticosteroid therapy as firstline therapy for chronic sinusitis. A short course of systemic corticosteroids (1-3 weeks), a short course of doxycycline (3 weeks), or a leukotriene antagonist may be considered in patients with nasal polyps. A prolonged cycle ( 3 months) of macrolide antibiotic can be considered for patients without polyps.

KEYWORDS chronic sinusitis, rhinosinusitis, diagnosis, treatment, antifungal, amphotericin B.

\section{INTRODUCCIÓN}

La sinusitis crónica ocupa el segundo lugar después de la artritis entre las enfermedades crónicas más comunes en las mujeres entre 45 y 65 años de edad (1) es una condición comúnmente diagnosticada en adultos que con frecuencia se presentan con enfermedad en etapa tardía y cambios irreversibles en la mucosa sinusal (2), que inhibe la función mucociliar de la nariz y los senos paranasales que dura más de 12 semanas. (3).

Entre las causas destacan la respuesta inflamatoria en las vías aéreas superiores secundaria a infección viral o bacteriana, Fúngica, los mediadores derivados de eosinófilos también podrían ser un factor importante para desarrollar inflamación nasal, Infección por Helicobacter pylori y reflujo laringofaríngeo. Se distinguen dos formas clínicas: rinosinusitis crónica con pólipos (Staphylococcus aureus y Haemophilus inluenzae entre las aerobias y Prevotella y Peptostreptococcus) y sin pólipos. Y según la topogrqafia las anteriores son la sinusitis maxilar Sinusitis etmoidal anterior Sinusitis frontal y las Posteriores Sinusitis etmoidal posterior Sinusitis esfenoidal; además monosinusitis y polisinusitis.

La sinusitis crónica refractaria es una enfermedad compleja de tratar en otorrinolaringología. Se han realizado muchas investigaciones sobre la patogénesis y el tratamiento de los senos crónicos refractarios. (4), en niños se produce por la inflamación 
crónica de los senos paranasales, especialmente los senos maxilares, con alergia respiratoria (5) y la incidencia de sinusitis asociada a infecciones odontógenas es muy baja a pesar de la alta frecuencia de infecciones dentarias ya que el suelo del seno tiene una cortical densa que es una barrera defensiva efectiva contra la infección. (6)

Por otro lado, la sinusitis aguda dura menos de 1 mes y los síntomas remiten completamente tiene lugar posterior a desarrollo una infección en las vías respiratorias superiores (7). Entre las causas de la sinusitis crónica se pueden incluir infecciones, crecimientos en los senos paranasales (pólipos nasales) o inflamación del revestimiento de los senos paranasales, también se denomina rinosinusitis crónica, que puede afectar tanto a adultos como a niños. (8).

La tomografía computarizada puede ayudar a los médicos a predecir la gravedad de los síntomas de obstrucción nasal y secreción, pero no de otros síntomas de rinosinusitis crónica. (9). Esta prueba puede proporcionar información acerca de la gravedad y localización. (10)

Los síntomas más comunes corresponden a nariz tapada o congestionada, secreción espesa de la nariz, presión o incomodidad en el rostro, reducción o falta del sentido del olfato o el gusto, si experimenta 2 o más de estos síntomas y duran más de 3 meses, es posible que tenga sinusitis crónica (10)

Los métodos diagnostico se basa en la historia clínica donde se indagará los antecedentes médicos y observará dentro de la nariz para ver cuál es el estado de la inflamación y el edema (10), requiere la existencia de signos y síntomas que pongan de manifiesto la afectación de los senos paranasales: dolor, secreción o sensibilidad del seno. (11), luego se realiza la endoscopia nasal, para observar en profundidad la nariz y los senos nasales, proporciona información acerca de diagnóstico y el tratamiento que podría necesitar. (10).

El tratamiento se basa en el trabajo interdisciplinario e incluye cirugía endoscópica funcional, realizada por el otorrinolaringólogo (12). El principal objetivo del tratamiento es reducir la inflamación y evitar las crisis, La reducción de las crisis puede mejorar su calidad de vida. La mayoría de las personas necesitan un tratamiento diario a largo plazo ("mantenimiento"). (10) Así también están indicadas los enjuagues con solución salina (lavados). En general se utilizan para eliminar la mucosidad, las bacterias y las partículas de la nariz y los senos nasales. (10). Esta revisión se centrará en el pensamiento actual y la evidencia de las opciones de tratamiento, incluido medidas de prevención.

\section{MATERIALES Y MÉTODOS}

Estudio descriptivo, documental cuyos datos fueron recolectados a través de fuentes secundarios en revistas indexadas, libros, sitios web publicados en los últimos cinco años 2015 al 2020 salvo los de relevancia científica de años previos, utilizando las palabras claves sinusitis crónica, rinosinusitis, etiología, tratamiento. Se escogió entre los idiomas el inglés y español se 
descartaron otros; seleccionado 25 artículos de los cuales 9 fueron imprescindibles y relevante para alcanzar el objetivo de esta investigación, donde detalla los diversos esquemas terapéuticos tanto farmacológicos como quirúrgicos. Los criterios de inclusión fueron artículos de revisión documental cuyo tema central fue terapéutica en pacientes con sinusitis crónica.

\section{RESULTADOS}

Las modalidades de tratamiento primario son los corticosteroides y los antibióticos con cirugía, una opción para fracasos, pero el nivel de evidencia de respaldo es generalmente bajo. La razón principal es que CRS es un complejo de síntomas y no una enfermedad específica (13).

Los inhibidores de interleukina 5 en pacientes con pólipos podrían disminuir el puntaje de pólipos nasales. Si bien podrían asociarse a efectos adversos, estos serían poco frecuentes y de baja severidad. Sin embargo, la certeza de la evidencia es baja (14).

La cirugía está indicada en pacientes con complicaciones orbitarias o intracraneales de rinosinusitis aguda refractarias al tratamiento farmacológico, rinosinusitis crónica (SRC) con infección sinonasal persistente y secreción purulenta, fibrosis quística, discinesia ciliar, dacriocistitis debido a sinusitis y resistente al tratamiento médico, rinosinusitis micótica.

El tratamiento simultáneo de las complicaciones intracraneales de la sinusitis es un método de tratamiento efectivo que tiene una carga mínima para el paciente (15)

El tratamiento de la sinusitis frontal crónica a través de la etmoidectomía es una alternativa potencial a la sinusotomía frontal, logrando mejoras similares en la calidad de vida (CV) en pacientes que manifiestan una enfermedad sinusal menos grave (16).

En relación a prevención se debe evitar el humo del tabaco y las toxinas ocupacionales. (17), minimizar el impacto de una enfermedad o lesión en curso que tiene efectos duraderos. Se ha demostrado que la ansiedad y la depresión están asociadas con la amplificación de los síntomas y pueden requerir tratamiento. (17)

La inmunoglobulina $E$ es un mediador inflamatorio que desempeña un papel etiopatogénico en esta afección y un anticuerpo monoclonal antiinmunoglobulina $\mathrm{E}$, podría ser una alternativa terapéutica. (18).

\section{DISCUSIÓN}

Se identificaron cinco revisiones sistemáticas que incluyeron cinco estudios primarios en general, de los cuales dos corresponden a ensayos aleatorios sobre la eficacia del omalizumab no queda claro si este fármaco conduce a una mejora en la escala de pólipos nasales, calidad de vida, bienestar general o síntomas nasales en estos pacientes, porque la certeza de la evidencia es muy baja.

Sin embargo, Joanne Rimmer concluye en su publicación que no existe evidencia clara de que un tratamiento sea mejor que otro en términos de puntajes de síntomas informados por el paciente y medidas de calidad de vida. El único hallazgo positivo de los diversos estudios que 
examinan varias comparaciones diferentes debe tratarse con la debida precaución, en particular cuando la importancia clínica de la medida es incierta. (19)

En un estudio de revisión en el año 2016 donde se evaluó los efectos de un ciclo corto de corticosteroides orales como terapia complementaria ("complemento") con tratamientos estándar, realizado por Jefe $\mathrm{K}$, et al donde concluye que no está claro si los beneficios de los corticosteroides orales como terapia complementaria se mantienen más allá del corto período de seguimiento informado (hasta 30 días), ya que no hubo datos a más largo plazo disponibles. Tampoco se encontró datos en esta revisión sobre los efectos adversos asociado con cursos cortos de corticosteroides orales como terapia complementaria. Más investigación en esta área. (20); Sin embargo Palme en el 2028 en su trabajo donde evaluó la seguridad y la eficacia de EDS-FLU 372 $\mu$ g dos veces al día en pacientes con SRC (con $[n=34]$ o $\sin [n=189$ ] pólipos nasales [NP] concluyo que durante más de 1 año de tratamiento en CRS con y sin NP, EDS-FLU $372 \mu \mathrm{g}$ dos veces al día fue bien tolerado y produjo mejoras en una amplia gama de medidas objetivas y subjetivas. EDS-FLU puede ser una nueva opción deseable para pacientes con esta afección (21).

En relación a los antibióticos tipos macrólidos Laso en el 2017 sobre macrólidos a dosis bajas concluye que no se puede llegar a una conclusión firme con respecto a la efectividad del uso de macrólidos con base en la evidencia disponible. Se necesita más estudio con un diseño controlado con placebo que evalúe el uso de macrólidos en estos pacientes. (22)

La cirugía sinusal sigue siendo un tema de discusión, si el tratamiento inicial falla, la cirugía endoscópica de seno de revisión es una opción. Las sinusotomías amplias permiten la visualización y el acceso a la enfermedad durante la operación. Las grandes aberturas sinusales también facilitan el desbridamiento postoperatorio en la clínica, el monitoreo de la enfermedad endoscópica y la terapia tópica de los senos (23). Entre las complicaciones se observan la lesión de la arteria etmoidal anterior, cuando se toma la decisión de someterse a una cirugía de revisión, el paciente y el médico deben comprender el programa riguroso y prolongado de atención postoperatoria y desbridamiento que pueden ser necesarios para el éxito a largo plazo. Debe respetarse la técnica quirúrgica apropiada que enfatiza la preservación de la mucosa y la disección completa (24), (25).

\section{CONCLUSIONES}

Entre la etiología de sinusitis crónica destacan la respuesta inflamatoria en las vías aéreas superiores secundaria a infección viral o bacteriana, fúngica, alergias, Infección por helicobacter pylori y reflujo laringofaríngeo.

El tratamiento de la sinusitis crónica comúnmente implica el uso de corticosteroides intranasales, antibióticos orales y cirugía. En el caso de la cirugía el medico que toma la decisión de realizar una cirugía, debe comprender el programa riguroso y prolongado para el éxito a largo plazo y la elección depende de realizar un diagnóstico correcto, requiere la revisión de 
los síntomas y de imágenes recientes, reevaluación de los factores ambientales, generales y locales del huésped que pueden contribuir a la enfermedad persistente.

Los corticosteroides reducen la inflamación de la mucosa sinonasal, disminuyen la permeabilidad vascular y reducen la liberación de glucoproteínas de las glándulas submucosas (es decir, mucosidad delgada). Los beneficios asociados con esta terapia tienen el mayor nivel de evidencia con 6 metanálisis que cuantifican la evidencia de más de 40 ensayos clínicos aleatorizados (ECA). Tres metanálisis con más de 3624 pacientes evaluados con pólipos nasales demostró una asociación con mejoras en los puntajes generales de los síntomas, el tamaño del pólipo y la tasa de recurrencia después de la cirugía sinusal (2).

Existen revisiones sistemáticas actualizadas donde concluyen que no existe firme evidencia con respecto a la efectividad del uso antibióticos, corticoides esto dependerá del factor de riesgo y etiología. Se necesita más estudio con un diseño controlado con placebo que evalúe de estos en poblaciones con sinusitis crónicas claramente definidas.

\section{REFERENCIAS}

1. Ivker RS. Sinusits cronica. In Ivert: Elsevier; 2009. p. 197-208.

2. Chang EH SDWAGSWAMF. Factores de riesgo de la vida temprana para la sinusitis crónica : un estudio de cohorte de nacimiento longitudinal. J Allergy Clin Immunol. 2018 Abril; 4: p. 1291-1297.

3. Morcom S PN,PA,TD. La sinusitis. Aust Fam Médico.. 2016 Junio; 6: p. 374-7.

4. Zhao CL YS. Avances clínicos recientes en sinusitis crónica refractaria. Lin Chung Er Bi Yan Hou Tou Jing Wai Ke Za Zhi. 2020 Enero; 1: p. 19-22.

5. GS Rachelefsky RKSS. Sinusitis crónica en el niño alérgico. Pediatr Clin North Am. ;: p. 1091101.

6. Grau AR. Evaluación radiográfica del seno maximilar incatvision. Estudio observacional retrospectivo de los factores etiológicos de las sinusitis maximilares. Universidad Complutense de Madrid. 2011 Septiembre 6.

7. Janet M. Torpy CLRMG. Sinusitis Crónica. La Revista de la American Medical Association. 2011;: p. 2048.

8. Mayo clinic. sinusitis cronica.; s/d [cited 2020 mayo 7. Available from: https://www.mayoclinic.org/es-es/diseases-conditions/chronic-sinusitis/symptomscauses/syc-20351661.

9. Enema Job Amodu AJFAOAADO. Rinosinusitis crónica: correlación de síntomas con hallazgos de tomografía computarizada. Pan Afr Med J. 2014 Mayo 10. 
10. La Hoja para el Paciente de JAMA. Sinusitis crónica en adultos. La Hoja para el Paciente de JAMA. 2015 Septiembre; 314(9).

11. A. Fernández PL. Sinusitis. Medicina General. 2000;: p. 761-770.

12. Kristine von Bischhoffshausen CTLSTCCCCHRSAVD. Diagnóstico y tratamiento de la sinusitis maxilar odontogénica. Rev. Otorrinolaringol. 2019; 79(3).

13. Ghogomu N KR. Rinosinusitis crónica : la justificación de los tratamientos actuales. Experto Rev Clin Immunol. 2017 Marzo; 3: p. 259-270.

14. María José Poblete ARMW. Terapia anti interleukina 5 para la rinosinusitis crónica con pólipos. Medwave. 2018; 6: p. e7300.

15. Szyfter W BABŁMAKZA. Tratamiento simultáneo de complicaciones intracraneales de sinusitis paranasal. Eur Arch Otorhinolaryngol. 2018 Mayo; 5: p. 1165-1173.

16. Abuzeid WM MJCMRLSZKGSTHP. Resultados de la sinusitis frontal crónica tratada con etmoidectomía: un estudio prospectivo. Int Forum Alergia Rhinol. 2016 Junio; 6: p. 597604.

17. Hopkins C SPBFHR,WAHP. Prevención de la rinosinusitis crónica. Rinología. 2018; 4: p. 307315.

18. Brañes R. Omalizumab para la rinosinusitis crónica. Medwave. 2018 noviembre; 18 (7).

19. Joanne Rimmer WFLYC,CH. Intervenciones quirúrgicas versus médicas para la rinosinusitis crónica con pólipos nasales. Cochrane, sist reviu. 2014 Diciembre 1.

20. Jefe K CLHCPCSABM. Esteroides orales de corta duración como terapia complementaria para la rinosinusitis crónica. Cochrane. 2016 Abril.

21. Palmer JN JKMJKGCDPMR. estudio de 1 año del sistema de entrega de exhalación con fluticasona (EDS-FLU) en la rinosinusitis crónica. Alergia Rhinol. .

22. Lasso A MPQJCJLVBJKS. Macrólidos a dosis bajas a largo plazo para la rinosinusitis crónica en adultos: una revisión sistemática de la literatura. Clin Otolaryngol.. 2017 Noviembre 25; 42(3).

23. Corinna G Levine RRC. Revisión Cirugía Endoscópica Funcional Sinusal. Otolaringol clin North Am. 2017 febrero; 50(1).

24. Guarnizo A. Evaluación de la tomografía computarizada de la dehiscencia del canal etmoidal anterior: un estudio de acuerdo interobservador y revisión de la literatura. Neuroradioloj. 2020 abr; 33 (2).

25. Cohen Na. Revisión Cirugía Endoscópica de Seno. Otolaryngol Clin. 2006 Junio; 39(3). 
http://www.jah-journal.com/index.php/jah 